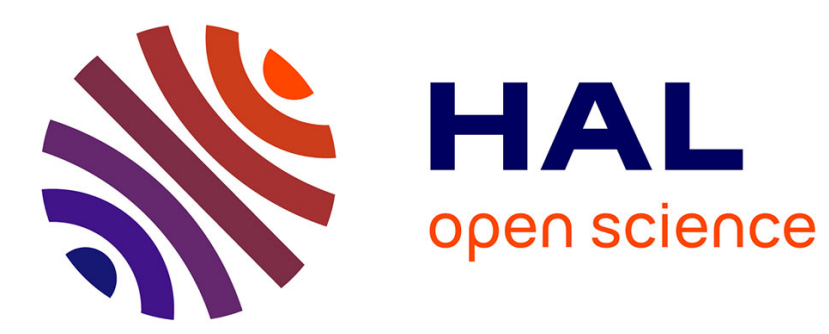

\title{
Effect of boundary conditions on the character of ambipolar diffusion in electrolytes
}

\author{
I. Chikina, V. B. Shikin, A. A. Varlamov
}

\section{To cite this version:}

I. Chikina, V. B. Shikin, A. A. Varlamov. Effect of boundary conditions on the character of ambipolar diffusion in electrolytes. Physical Review E : Statistical, Nonlinear, and Soft Matter Physics, 2015, 92 (1), pp.012310. 10.1103/PhysRevE.92.012310 . hal-01187805

\section{HAL Id: hal-01187805 \\ https://hal.science/hal-01187805}

Submitted on 17 Nov 2015

HAL is a multi-disciplinary open access archive for the deposit and dissemination of scientific research documents, whether they are published or not. The documents may come from teaching and research institutions in France or abroad, or from public or private research centers.
L'archive ouverte pluridisciplinaire HAL, est destinée au dépôt et à la diffusion de documents scientifiques de niveau recherche, publiés ou non, émanant des établissements d'enseignement et de recherche français ou étrangers, des laboratoires publics ou privés. 


\title{
Effect of boundary conditions on the character of ambipolar diffusion in electrolytes
}

\author{
I. Chikina, ${ }^{1}$ V. B. Shikin, ${ }^{2}$ and A. A. Varlamov ${ }^{3}$ \\ ${ }^{1}$ IRAMIS, LIONS, UMR NIMBE 3299 CEA-CNRS, CEA-Saclay, F-91191 Gif-sur-Yvette Cedex, France \\ ${ }^{2}$ ISSP, RAS, Chernogolovka, Moscow District 142432, Russia \\ ${ }^{3}$ CNR-SPIN, Viale del Politecnico 1, I-00133 Rome, Italy
}

(Received 26 February 2015; published 15 July 2015)

\begin{abstract}
We discuss the details of ambipolar relaxation of the electric field in liquid asymmetric electrolytes to its stationary value. It is demonstrated that the account for finite boundary conditions modifies the existing concepts of this diffusion process. In particular, we succeeded to suggest a qualitatively correct explanation of the observed distribution of the electric fields over the bulk of the cuvette and its nonmonotonic behavior in measurements on the finite-size cuvette. We analyze the conditions of such an anomaly at the intermediate stages of the relaxation process.
\end{abstract}

DOI: 10.1103/PhysRevE.92.012310

PACS number(s): 66.10.C-, 47.57.ef

\section{INTRODUCTION}

Ambipolar diffusion is the process of mutual diffusion of positive and negative charge carriers in the environment of prevailing neutral particles in the presence of the local carriers density gradients $\nabla n_{ \pm}$. Evidently, the two sorts of carriers do not move independently: the initially fast motion of the more mobile particles causes the formation of a charge imbalance. The electric field arising due to such local charge separation results in particle interaction, which plays the essential role in the process of ambipolar diffusion [1]. Due to this field, more mobile particles drag less mobile ones against the density gradients.

The characteristic feature of ambipolar diffusion is the quasineutral behavior of the whole system in the stationary regime in the case in which the single-component diffusion coefficients $\mathcal{D}_{ \pm}$differ strongly $\left(\mathcal{D}_{+} \gg \mathcal{D}_{-}\right)$. Due to the Einstein relation $\mathcal{D}_{ \pm}=k_{B} T \chi_{ \pm}$(see, for example, Ref. [1]; here $k_{B}$ is the Boltzmann constant, which we will assume below to be equal to 1), this requirement also implies a strong difference in the carrier mobilities: $\chi_{+} \gg \chi_{-}$. The value of the internal electric field can be obtained in this case in the assumption of the smallness of the carrier flow and density imbalances. In the presence of an electric field and a density gradient, the charge carrier flows $j_{ \pm}$are given by the sum of a drift and a diffusion component. The former is driven by the electric field, $j_{ \pm}^{\text {(drift) }}=\mp \chi_{ \pm} n_{ \pm} E$, while the latter is driven by the gradient of concentration, $j_{ \pm}^{\text {(diff) }}=-\mathcal{D}_{ \pm} \nabla n_{ \pm}$. Equating $j_{+} \simeq j_{-}$and $n_{+} \simeq n_{-}$, one obtains the expression for the internal electric field occurring due to the local break of electroneutrality and preventing the global charge separation:

$$
E=-\varphi_{x}^{\prime}=\left(\frac{\mathcal{D}_{+}-\mathcal{D}_{-}}{\chi_{-}+\chi_{+}}\right) \frac{\nabla n}{n},
$$

where $n(x, t)$ is the local value of particle density (time dependence takes place when the system relaxes to the state of stationary diffusion). As a result, the neutral in average mass current $j=-\mathcal{D}_{a} \nabla n$ in the ambipolar approximation is determined by the effective diffusion coefficient

$$
\mathcal{D}_{a}=\frac{2 k_{B} T}{\chi_{-}^{-1}+\chi_{+}^{-1}}=k_{B} T \chi_{a} .
$$

Instead of $\mathcal{D}_{a}$, one can use the effective mobility $\chi_{a}$. The corresponding ambipolar resistivity is determined as the average of the charge carrier resistivities: $\chi_{a}^{-1}=\left(\chi_{+}^{-1}+\chi_{-}^{-1}\right) / 2$.

Equations (1) and (2) indicate that the flows of the charged particles of the opposite signs in the system under consideration cannot exist independently. As soon as the difference $n_{+}-n_{-}$emerges at some domain, the electric field (1) arises, which counteracts the further growth of the density imbalance. As a result, the ambipolar transport remains quasineutral: its two-component nature shows up itself only in the definition of the effective diffusion coefficient (2).

The kinetics of plasma [2] and the transport phenomena in gases [3], semiconductors [4], electrolytes [5,6], and ionic crystals [7] are the traditional problems dealing with transport of oppositely charged flows. The ambipolar approach could also be applied to the analysis of the self-consistent system of equations in the theory of the Seebeck effect in electrolytes [8-11]. The role of ambipolar specifics can be important in relation to the Einstein rules in nonequilibrium thermodynamics $[12,13]$. The ambipolar mechanism of the protostar formation is discussed in astrophysics [14,15], etc. At the same time, the universality of the ambipolar simplifications is far from evident, in spite of the existence of a variety of their applications. In particular, the question of sensitivity of the ambipolar approximation to the boundary and the initial conditions, which always arises in the transport problems, remains unanswered.

Let us illustrate the validity of Eqs. (1) and (2) with a simple example in which, at the initial moment $t=0$, both positive and negative ions are collected at the same point $x=0$, i.e., their initial density distributions have the form of a $\delta$ function. The particles of both subsystems start to diffuse with corresponding mobilities $\chi_{-}$and $\chi_{+}$, and more mobile particles initially outrun the slower ones. Nevertheless, the internal electric field (1) is formed due to the spacecharge separation, and after some time the system passes into the regime of the ambipolar diffusion. The corresponding relaxation time can be evaluated [1] as the one required for diffusion of the light particles on the distance of the Debye screening: $\tau_{a} \propto a_{D}^{2} / \max \left\{\mathcal{D}_{+}, \mathcal{D}_{+}\right\}$. Further motion of two subsystems occurs in a coordinated way with the unique diffusion coefficient (2), and the density distribution acquires 
the standard exponential form:

$$
n\left(x, t \gg \tau_{a}\right) \propto \frac{1}{2 \sqrt{\pi \mathcal{D}_{a} t}} \exp \left(-\frac{x^{2}}{4 \mathcal{D}_{a} t}\right) .
$$

The natural boundary conditions for the discussed example read $n(x, t=0) \propto \delta(x)$ and $n( \pm \infty, t)=0$. One can easily see that they are compatible with Eqs. (1) and (2).

Let us stress that Eq. (3) does not provide us with any characteristic length: as a result of diffusion, particles can reach any point $x$ after the time $\sim x^{2} / \mathcal{D}_{a}$ from the beginning of diffusion. At the same time, in the real problems of diffusion, the boundary conditions are applied at finite length, for instance at the borders of the cuvette in the problem of diffusion in electrolytes. Below we will demonstrate that the standard ambipolar diffusion description in terms of Eqs. (1) and (2) is valid until the characteristic lengths of the spacial inhomogeneity due to the boundary conditions remain much larger than the lengths of the particle localization in the external field. To provide a representative example, we start our discussion with the problem of sedimentation of two subsystems of the charged particles with different masses in the gravitation field with semi-infinite boundary conditions. Then we will discuss the problem of ambipolar type with finite boundary conditions, addressing the relaxation of the electric field in the Seebeck effect in electrolytes. Possible applications of the proposed theory are discussed in the Conclusion.

\section{AMBIPOLAR DIFFUSION IN THE PROBLEM WITH THE SEMI-INFINITE BOUNDARY CONDITIONS}

Let us check the applicability of the simple "recipe" given by Eqs. (1) and (2) to the problem of ambipolar diffusion with the semi-infinite boundary conditions. In particular, we will consider the neutral in whole asymmetric electrolyte (two subsystems of charged particles of different masses $M \gg m$ and the opposite charges) with the initial $\delta$-function distribution of its concentration at the point $y=L>0$ placed in gravitational field.

The consideration of the two-component system in ambipolar diffusion regime and the validity of this approximation we will precede by the discussion of the case of one-component system of neutral particles with the semi-infinite boundary conditions in external field. The diffusion equation has been enriched by taking into account the gravitational component, while the boundary conditions correspond to the initial $\delta$ function distribution of the concentration and zero mass flow through the bottom of the volume:

$$
\begin{aligned}
\frac{\partial n}{\partial t} & =\mathcal{D} \frac{\partial^{2} n}{\partial y^{2}}-s \frac{\partial n}{\partial y}, \\
n(y, L, t \rightarrow 0) & \rightarrow \delta(y-L), \\
\mathcal{D} \frac{\partial n}{\partial y}+\left.s n\right|_{y=0, t>0} & =0
\end{aligned}
$$

with $s=m \chi g$. The first term on the right-hand side of this equation accounts for the diffusion, the second corresponds to the "fall down" of the particles in gravitational field.
To analyze this equation, we first perform the following transformation of the variable:

$$
n(y, t, L)=N(y, t, L) \exp \left[-\frac{s(y-L)}{2 \mathcal{D}}-\frac{s^{2} t}{4 \mathcal{D}}\right],
$$

which reduces Eq. (4) to the standard form

$$
\frac{\partial N}{\partial t}=\mathcal{D} \frac{\partial^{2} N}{\partial y^{2}} \text {. }
$$

In this representation, the smearing of the initial distribution of neutral particles is described by Eq. (5) while the drift of the concentration distribution peak follows from the condition

$$
y_{\max }(t)=L-s t / 2 .
$$

The exact solution of Eq. (4) takes the form [16]

$$
\begin{aligned}
n(y, t, L)= & \frac{1}{2 \sqrt{\pi \mathcal{D} t}}\left\{\exp \left[-\frac{(y-L)^{2}}{4 \mathcal{D} t}\right]+\exp \left[-\frac{(y+L)^{2}}{4 \mathcal{D} t}\right]\right\} \\
& \times \exp \left[\frac{(L-y)}{2 \ell_{B}}-\frac{\mathcal{D}_{a} t}{4 \ell_{B}^{2}}\right] \\
& -\frac{1}{2 \ell_{B}} \exp \left(-\frac{y}{\ell_{B}}\right)\left[1-\operatorname{erf}\left(\frac{y+L}{2 \sqrt{\mathcal{D} t}}-\frac{\sqrt{\mathcal{D} t}}{2 \ell_{B}}\right)\right],
\end{aligned}
$$

where

$$
\operatorname{erf}(x)=\frac{2}{\sqrt{\pi}} \int_{0}^{x} e^{-z^{2}} d z
$$

is the error function.

One can see that the found particles density distributions for the cases "1"-" 6 " have the well pronounced maxima moving (falling down) in the direction of the wall. Starting from the case "7" and up to "10," a stationary regime is being established, which corresponds to the formation of the the characteristic length of the Boltzmann distribution $\ell_{B}=T /(m g)=0.1 L$.

The dependence of the positions of the distribution maximum (7) as the function of time is presented in Fig. 1. The linear part of this dependence corresponds to the validity of the approximations of Eqs. (4)-(6). The nonlinearity shows up itself when the position of this maximum approaches the wall (i.e., at the distances of the order of $\ell_{B}=0.1 L$ ).

Let us return to the two-component system with ions of the different masses $M \neq m$, mobilities $\chi_{+} \neq \chi_{-}$, and charges $e_{M}=-e_{m}$. It is possible now to formulate the conditions under which the solution of Eq. (4) in the form (7) is valid for such a system (ambipolar approximation). This approximation is justified when Eq. (6) is satisfied. The definition of the velocity $s$ in the latter should include the minimal of the mobilities, and under the diffusion coefficient $\mathcal{D}$ in Eq. (5) one should imply the coefficient of ambipolar diffusion $\mathcal{D}_{a}$ [see Eq. (2)]. The considered two-component system will demonstrate regimes "1"-“7" (see Fig. 2) when all this assumptions are fulfilled.

The ambipolar regime fails for longer times (regimes " 8 "" $10 ")$. In the vicinity of the impenetrable potential wall $(y=0)$ the kinetics of the two-component system has to be considered without the ambipolar approximation. In the next subsection we will proceed to discussion of this regime. 


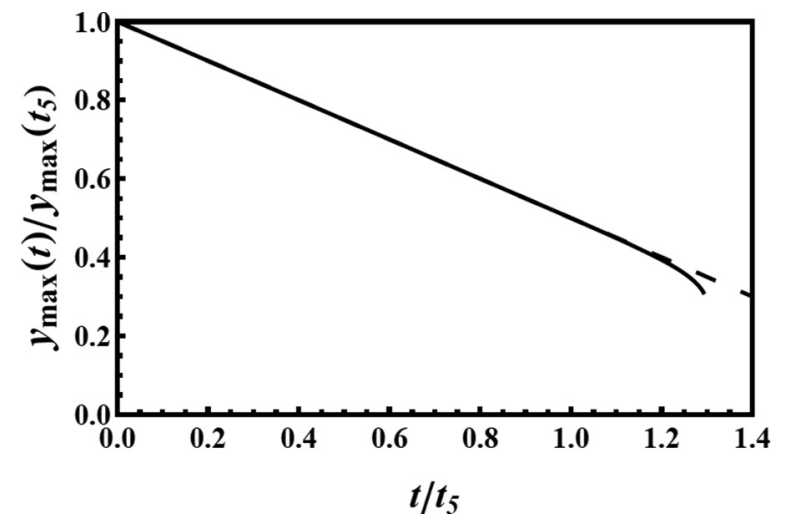

FIG. 1. The dimensionless dependence $y_{\max }(t) / y_{\max }\left(t_{5}\right)$ following from the numerical solution of Eq. (6).

\section{FINAL STAGE OF THE ION DENSITY RELAXATION}

We are speaking about the final stage of the ions density relaxation in the asymmetric electrolyte with very different lengths $l_{m} \gg L_{M}=T / M g \ll L$ and the opposite charges of the particles $\pm e$. Here $L$ is the length of the system in the direction of gravitational field which is supposed to be much

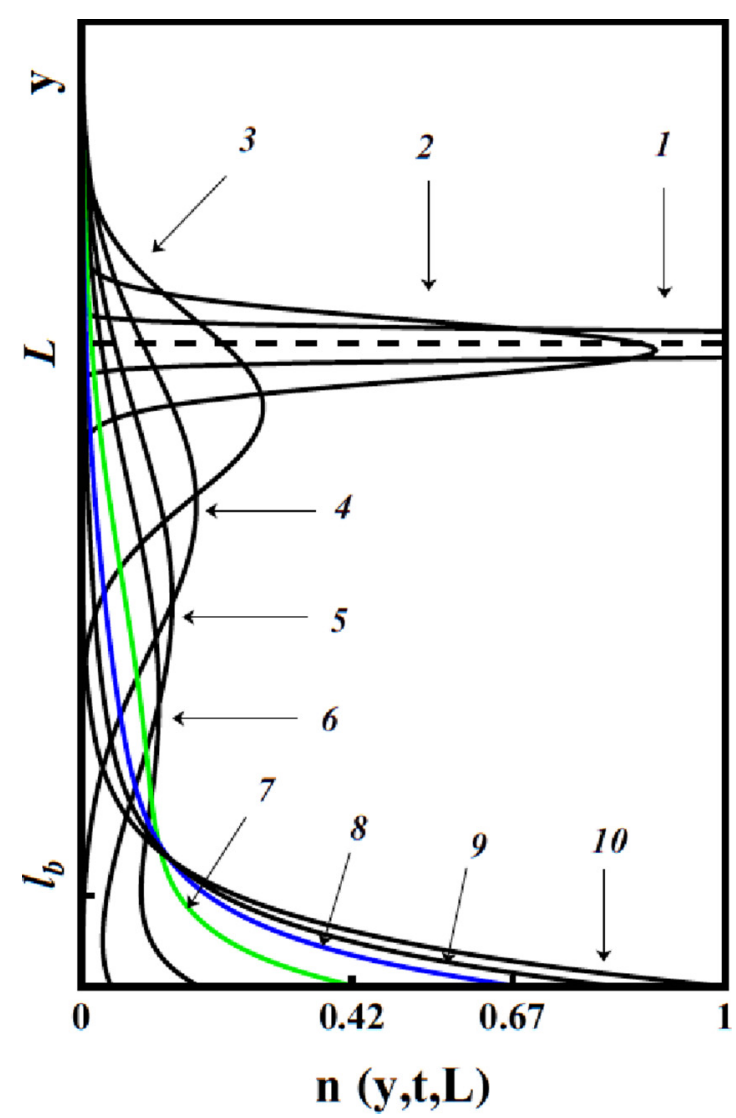

FIG. 2. (Color online) Evolution of the density of particles $n(y, L, t)$ for $\ell_{B} / L=0.1\left(\ell_{B}=1, L=10\right)$ and $t=0.01,0.1,1$, $2.5,4,5.5,7.5,10,12$, and 100 in the problem of diffusion with the semi-infinite boundary conditions. The initial density distribution is chosen in the form of the $\delta$ function at the point $y=L$, located far enough from the impenetrable wall $(y=0)$. larger than both lengths $l_{m}$ and $L_{M}$. The difference of $l_{m}$ and $L_{M}$ results in the charge separation and formation in electrolyte of the internal electric field $E$, which, in its turn, affects on ion density distribution. The lengths become dependent on the value of this field [17]:

$$
\tilde{l}_{\mathrm{m}}(E)=T /[m g+|e| E], \quad \tilde{L}_{\mathrm{M}}(E)=T /[M g-|e| E] .
$$

Consequently, the Boltzmann density distribution of the light particles

$$
\tilde{n}(x) \propto n_{i}\left(\frac{L}{\tilde{l}_{\mathrm{m}}}\right) \exp \left[-\frac{x}{\tilde{l}_{m}}\right]
$$

shrinks, while that one of heavy particles

$$
\widetilde{N}(x) \propto n_{i}\left(\frac{L}{\widetilde{L}_{\mathrm{M}}}\right) \exp \left[-\frac{x}{\tilde{L}_{M}}\right]
$$

stretches. Here $n_{i}$ is the density of ions in absence of gravitational field. The supreme field $E^{*}$, determined by the condition $\tilde{l}_{m}\left(E^{*}\right)=\widetilde{\widetilde{L}}_{M}\left(E^{*}\right)$, restricts the limits of applicability of the model:

$$
E^{*}=\frac{g}{2 e}(M-m) .
$$

Charge separation can be modeled as the double layer. The intensity of corresponding electric field, induced within the volume of this double layer, is expressed in terms of $E^{*}$ and the characteristic "interaction constant" $\zeta$,

$$
E=\frac{\zeta}{1+\zeta} E^{*}, \quad \zeta=\frac{8 \pi e^{2} n_{i} L}{\epsilon g M} .
$$

The explicit expressions for the renormalized lengths take the form $(m \ll M)$

$$
\tilde{l}_{\mathrm{m}}(\zeta)=l_{\mathrm{m}} \frac{1}{1+\frac{M}{2 m} \frac{\zeta}{1+\zeta}}, \quad \tilde{L}_{\mathrm{M}}(\zeta)=L_{\mathrm{M}} \frac{1+\zeta}{1+\zeta / 2} .
$$

In the limit of strong interaction,

$$
\tilde{l}_{\mathrm{m}}(\zeta \gg 1) \rightarrow \tilde{L}_{\mathrm{M}}(\zeta \gg 1)=2 L_{\mathrm{M}}
$$

both lengths are equal to each other. The internal electric field at this point reaches its maximal value $E^{*}$ [see Eq. (11)].

The transition of the Boltzmann-like picture of the neutral stationary state with the independent $l_{m}$ and $L_{M}$ to the mixed scenario described by Eqs. (8)-(14) was observed while experimentally [18] studying the electrolyte with $M / m \simeq 10$ in the field of centrifugal forces, which played the role of gravitation. The authors detected appearance of the potential difference between the endpoints of the test-tube. They attributed this Seebeck-like field to the effect of centrifugal forces acting differently on the diverse kinds of ions of the electrolyte.

The computer simulation of the equilibrium sedimentation density profiles of charged binary colloidal suspensions were performed in Ref. [19] in the frameworks of the densityfunctional theory. The sedimentation-diffusion equilibrium of dilute binary, ternary, and polydisperse mixtures of colloidal particles with different buoyant masses and/or charges was studied by means of Monte Carlo simulations in Refs. [20,21]. The formation of the inhomogeneous macroscopic electric field similar to Eq. (12) was found. The authors stressed that 
resulting electric force lifts the colloids against gravity, yielding highly nonbarometric and even nonmonotonic colloidal density profiles, which closely agrees with our conclusions.

The above example demonstrates how the ambipolar approximation fails close to the impenetrable wall. Indeed, in accordance to Eqs. (1) and (7), the maximum of the electric field should be located at the boundary $x=0$. In reality the distribution of the electric field determined by Eqs. (8)-(13) is much more sophisticated: its maximal value is definitely shifted in depth of nonhomogeneous electrolyte for the distance

$$
x_{0} \sim \tilde{L}_{M} \ln \left(\tilde{l}_{m} / \tilde{L}_{M}\right),
$$

where $\tilde{l}_{m}$ and $\tilde{L}_{M}$ are defined by Eq. (13).

\section{AMBIPOLAR RELAXATION IN THE SMALL DOMAIN AND ITS MANIFESTATION IN THE SEEBECK EFFECT}

\section{A. Breakdown of the ambipolar diffusion paradigm}

The anticipated generality of Eqs. (1) and (2) allows us to use them in order to describe the Seebeck effect in the ambipolar electrolyte. The latter consists of the emergence of the potential difference between the electrodes located at the extremes of the cuvette $( \pm L / 2)$ filled by electrolyte subjected to a temperature gradient (see Fig. 3). The inhomogeneous concentration distribution of the neutral particles $n_{i}(x, t)$ of the sort $i$ can be chosen as the solution of the diffusion equation describing the evolution of the initially homogeneous distribution $n_{i}(x, t=0)=$ const to the stationary one with the constant gradient $d n_{i}(x, t) /\left.d x\right|_{\forall x, t \rightarrow \infty}=$ const at the end of the process (see Ref. [22]):

$$
n_{i}(x, t)=\frac{\mathcal{D}_{T}^{(i)}}{\mathcal{D}^{(i)}} \nabla_{x} T\left[x-\frac{4}{\pi^{2}} \sum_{l=1}^{\infty} \frac{\sin ^{2} \frac{\pi l}{2}}{l^{2}} \sin (\pi l x) e^{-\frac{l^{2} t}{\tau_{i}}}\right] .
$$

Here $x \in[-L / 2, L / 2] \mathcal{D}^{(i)}$ and $\mathcal{D}_{T}^{(i)}$ are the diffusion and thermodiffusion coefficients, and $\tau_{i}=L^{2} /\left(\pi^{2} \mathcal{D}^{(i)}\right)$ is the characteristic time of the establishment of the stationary solution for the particles of the sort $i$. The behavior of the function $n_{i}(x, t)$ for different time intervals is shown in Fig. 4.

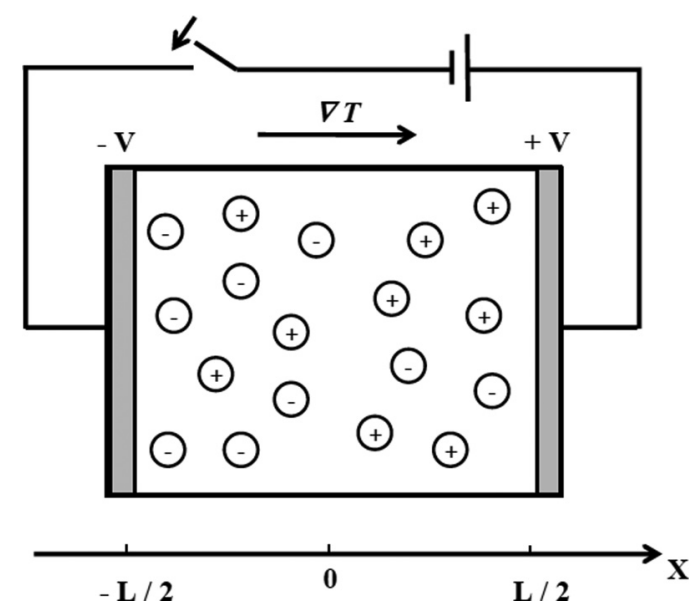

FIG. 3. The sketch of the experimental setup for measurements of the Seebeck effect.

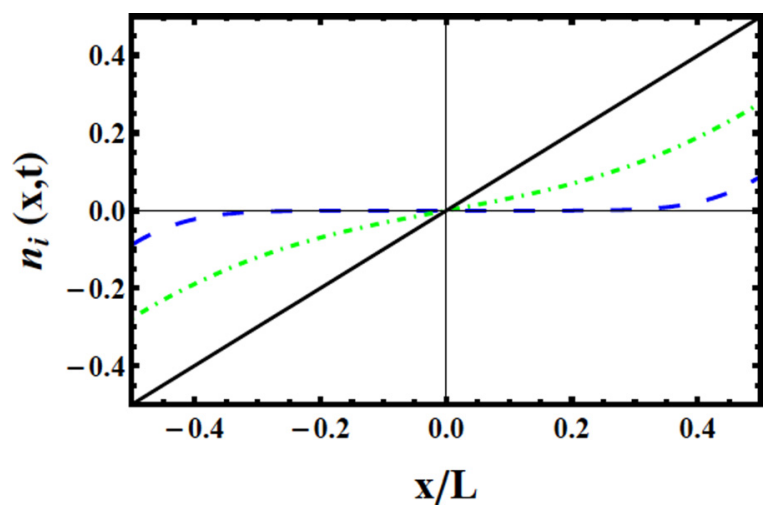

FIG. 4. (Color online) The relative behavior of $n_{i}(x ; t)$ (16) for different times $t_{1} / \tau_{i}=0,3$ (dashed line), $t_{2} / \tau_{i}=3$ (dot-dashed line), and $t_{3} / \tau_{i}=30$ (solid line). The last of these distributions corresponds to the stationary Soret effect.

The last of these distributions corresponds to the stationary Soret effect.

One could try to determine the Seebeck electric field based on Eq. (1) ignoring the internal Coulomb forces and using the concentration distribution (16). Unfortunately, defined in this way, the Seebeck field $E_{x}(t)$ has a qualitatively wrong time evolution. Indeed, during almost the entire transition period from the constant concentration to the constant gradient of concentration (see Fig. 4), $d n(x, t) / d x$ remains maximal in the vicinity of electrodes (see Fig. 3), close to the borders of the cuvette, being minimal in its central part. The corresponding electric field (1) is distributed similarly to the gradient (which we assume to be small), and this is in striking contrast with the experimental findings [23].

The origin of the profound discrepancy between the results (1), (2), and (16) and the experiment is hidden in the abovementioned restrictions of the validity of the ambipolar diffusion paradigm. The estimation of the characteristic Boltzmann length for the temperature field in conditions of the experiment [23] turns out to be much larger than the cuvette size, which makes the ambipolar approximation inapplicable; the process of electric-field relaxation becomes strongly dependent on the boundary conditions. Since the exact solution of the problem is unknown below, we will propose an alternative to Eqs. (2), (1), and (3).

\section{B. Ambipolar relaxation determined by the boundary conditions}

As we just discussed above, the transition of a onecomponent system of the neutral particles from the homogeneous state with $n_{i}(x, t=0)=$ const to the stationary state with $d n_{i}(x, t) /\left.d x\right|_{\forall x, t \rightarrow \infty}=$ const subjected to the temperature field $d T(x, t) /\left.d x\right|_{\forall t,}=$ const $(x \in[-L / 2, L / 2])$ is determined by Eq. (16). In the case of a two-component system of neutral particles different responses of the different particle types to a temperature gradient result in the effect of thermophoresis (the Soret effect). The Seebeck effect occurs when the temperature gradient is applied to the two-component electrolyte with the ions of the opposite charges and different diffusion (or thermodiffusion) coefficients. The emerging difference 


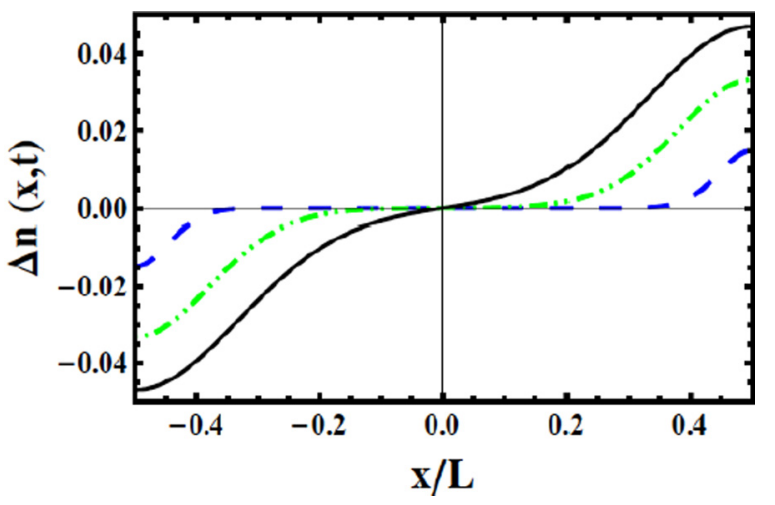

FIG. 5. (Color online) The spatial distribution of the charge separation $\Delta n(x, t)$ [see Eq. (17)] for different times $t_{1} / \tau_{+}=0.1$ (dashed line), $t_{2} / \tau_{+}=0.5$ (dot-dashed line), and $t_{3} / \tau_{+}=1.0$ (solid line) $\left(\tau_{-} / \tau_{+}=0.5\right.$; the relative Soret coefficient is equal to 0.1$)$.

between the mass flows of the diverse components results in the formation of the local charge separation,

$$
q \Delta n(x, t)=q\left[n_{+}(x, t)-n_{-}(x, t)\right] .
$$

The Coulomb forces arising due to this separation in their turn influence the relaxation process.

The time evolution of the spacial distribution of the charge imbalance (17) is shown in Fig. 5. It is seen that the Seebeck field is formed mainly due to the redistribution of the particles in the domains located close to the borders of the cuvette. This is why, in order to determine its shape, one can use the solutions (16) written for positive and negative ions with the correct boundary conditions. The difference between the positive and the negative ion concentrations $\Delta n(x, t)$ is evidently the odd function of $x$. One can notice that at the early stages of the relaxation process, the function $\Delta n(x, t)$ is localized in the domains close to the cuvette border, tending to become the linear function over the whole cuvette volume at times.

The next step is to introduce the effective surface charge density,

$$
\sigma(t)=q \int_{0}^{L / 2} \Delta n(x, t) d x .
$$

The Seebeck electric field now can be determined as the homogeneous field of the plane capacitor:

$$
E_{S}(t)=\frac{4 \pi \sigma(t)}{\kappa},
$$

where $\kappa$ is the dielectric constant of the solution.

Here we used a characteristic feature of the threedimensional Poisson equation, namely the existence of the homogeneous electric field in the conducting media without breaking the homogeneity of the surface charge distribution at the capacitor plates. This particular property justifies the simplification (18) and allows us to avoid a direct solution of the Poisson equation with the true charge distribution arising as the difference of the corresponding densities of ions [Eq. (16)].

The proposed scheme results in a qualitative agreement of the Seebeck field obtained in this way with the experimental findings. First of all, its space structure is correct: the maximum

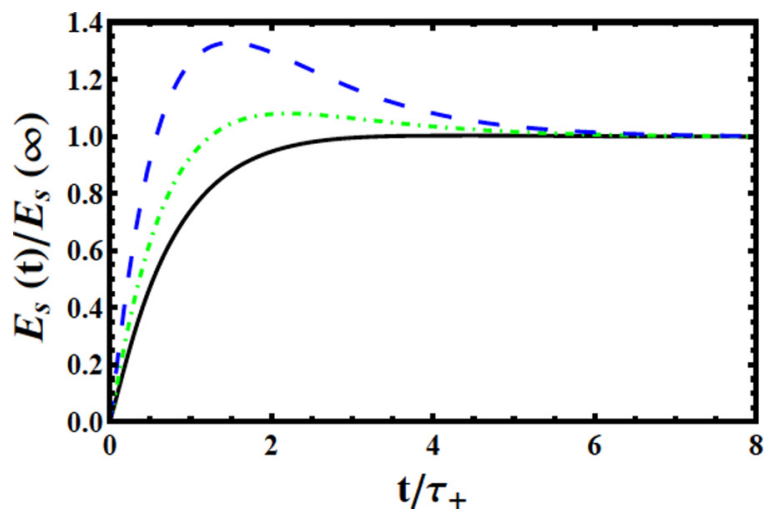

FIG. 6. (Color online) The behavior of the Seebeck electric field (19) as a function of time for the various fixed differences of the thermal diffusion coefficients and different relative values of the relaxation times of positive and negative ion systems $\tau_{+}$and $\tau_{-}$. The time scale is normalized to the largest relaxation time $\tau_{+}$, while the value of the Seebeck field is normalized to its value at $t=\infty$. The parameters corresponding to the dashed, dot-dashed, and solid lines are $\left(\tau_{+}-\tau_{-}\right) / \tau_{+}=0.02587 ; 0.07142 ; 0.14286$.

is located now in the central part of the cuvette instead of being close to the extremes of the cuvette [as would follow from Eqs. (1) and (16)].

Another essential property of the solution (19) is its possible nonmonotonic behavior as a function of time (see Fig. 6). The Seebeck electric field evidently starts its rise from zero $E_{S}(t=0)=0$. It is also clear that this field saturates at large times, $E_{S}(t \rightarrow \infty)=$ const This relaxation is definitely monotonous, i.e., $d E_{S}(t) / d t \geqslant 0$, in the particular case $\tau_{+}=$ $\tau_{-}$. The character of $E_{S}(t)$ relaxation to its final value in the general case $\tau_{+} \neq \tau_{-}$cannot be predicted in detail in the framework of the ambipolar diffusion paradigm. The proposed scheme shows a deficiency of the handmade accounting for the Coulomb interaction between the positive- and negativeion subsystems. Nevertheless, our numeric analysis of this relaxation process allows us not only to demonstrate the existence of the nonmonotonic regimes for certain relations between $\tau_{+}$and $\tau_{-}$, but also to study the dependence of the maximum position on the difference $\tau_{+}-\tau_{-}$. The appearance of such a maximum is completely counterintuitive, but it corresponds to the available experimental findings [23].

\section{CONCLUSIONS}

The above study of the ambipolar relaxation of the Seebeck electric field in the electrolyte to its stationary value demonstrates the importance of the role of boundary conditions. We have shown that the standard scheme described by Eqs. (1) and (2) remains valid until the diffusion of charged particles is considered in the large cuvette, not too close to its borders. The geometric size of the system for the validity of such consideration should noticeably exceed the characteristic length of the Boltzmann distribution. The latter can have an arbitrary origin, being thermal, gravitational, Hall-like, etc. In the opposite case, the account for finite boundary conditions becomes crucial, modifying the existing conception of the diffusion processes. We proposed an alternative to the 
approximation in Eqs. (1) and (2) in which framework we discuss the details of the electric-field distribution over the volume of the electrolyte in the cuvette. Among the results obtained is the formal confirmation of the maximum of the Seebeck field in the central part of the cuvette, instead of its periphery, as it follows from Eqs. (1) and(2). Our approach also allows us to describe, at least qualitatively, the evolution of the electric field measured in the bulk of the finite-size cuvette as a function of time. It is remarkable that we have succeeded to obtain its nonmonotonic behavior (see Fig. 6), which was observed in the experiment [23]. The standard approach in no way allows us to explain such a nontrivial experimental finding.

It is also worthwhile to emphasize the possibility of the geophysical applications of Eqs. (8)-(14). Let us evaluate the consequences of the proposed theory to the water vapor existing in Earth's atmosphere. The water molecule $\mathrm{H}_{2} \mathrm{O}$ dissociates to the proton $\mathrm{H}^{+}$and the hydroxyl group $\mathrm{OH}^{-}$. These ions form clusters due to the ion dipole interaction with the surrounding solvent. The effective masses of these formations are significantly different. Both types of ions are subject to the gravitational field and Coulomb interactions, i.e., corresponding low ionized plasma can be described by Eqs. (8)-(14). In accordance with Eq. (8), the characteristic lengths of the proton and hydroxil Boltzmann distributions are renormalized, and the Seebeck field (11) appears in the perturbed domain. The corresponding length for a proton with mass $m_{p} \approx 5 \times 10^{-24} \mathrm{~g}$ for the temperature of ambient $l_{m} \sim 100 \mathrm{~km}$, while for the hydroxyl group $\mathrm{OH}^{-}$this value is only $l_{\mathrm{OH}^{-}} \sim 6 \mathrm{~km}$. Therefore, at the heights of $10-100 \mathrm{~km}$, one could expect the existence of the steady ionized layer of the components of the water dissociation. Equations (8)-(14) indicate the formation of such ionized layers in the atmosphere due to the products of dissociation of different molecules. The existence of such layers is empirically well known [24,25], but, to the best of our knowledge, there has never been a reasonable explanation for them.

\section{ACKNOWLEDGMENT}

The authors acknowledge the support of the FP7-IRSES program, Grant No. 236947 "SIMTECH."
[1] E. M. Lifshitz and L. P. Pitaevskii, Physical Kinetics (Pergamon, New York, 1981).

[2] A. Akhiezer et al., Plasma Electrodynamics (Pergamon, New York, 1975).

[3] A. Nedospasov, Sov. Phys. Usp. 11, 174 (1968).

[4] W. Shockley, Electrons and Holes in Semiconductors, with Applications to Transistor Electronics (D. Van Nostrand company, Inc., Princeton, NJ, 1950).

[5] V. G. Levich, Physicochemical Hydrodynamics (Prentice-Hall, Englewood Cliffs, NJ, 1962).

[6] S. de Groot and P. Mazur, Non-equilibrium Thermodynamics (North-Holland, Amsterdam, 1962).

[7] J. Manning, Diffusion Kinetics for Atoms in Crystals (Van Nostrand, Princeton, 1968).

[8] A. Wurger, Phys. Rev. Lett. 101, 108302 (2008).

[9] A. Wurger, Phys. Rev. Lett. 102, 078302 (2009).

[10] J. Morthomas and A. Wurger, Europhys. J. E 27, 425 (2008).

[11] A. Wurger, Rep. Prog. Phys. 73, 126601 (2010).

[12] H. Zhao, Appl. Phys. Let. 92, 112104 (2008).
[13] A. Efros, Phys. Rev. Lett. 104, 116404 (2010).

[14] T. Mouschovias, Astro. Phys. J. 373, 169 (1991).

[15] S. Basu and T. Mouschovias, Astrophys. J. 432, 720 (1994).

[16] S. Chandrasekhar, Rev. Mod. Phys. 15, 1 (1943).

[17] I. Chikina, V. B. Shikin, and A. A. Varlamov, Phys. Rev. E 86, 011505 (2012).

[18] M. Rasa and A. Phillips, Nature (London) 429, 857 (2004).

[19] A. Esztermann and H. Löwen, Europhys. Lett. 68, 120 (2004).

[20] R. van Roij, J. Phys.: Condens. Matter 15, S3569 (2003).

[21] J. Zwanikken and R. van Roij, Europhys. Lett. 71, 480 (2005).

[22] J. Agar and J. Turner, Proc. R. Soc. London, Ser. A 255, 307 (1960).

[23] M. Bonetti, S. Nakamae, M. Roger, and P. Guenoun, J. Chem. Phys. 134, 114513 (2011).

[24] J. K. Hargreaves, The Upper Atmosphere and Solar-Terrestrial Relations (Cambridge University Press, Cambridge, 1992).

[25] M. C. Kelley and R. A. Heelis, The Earth's Ionosphere: Plasma Physics and Electrodynamics (Academic Press, San Diego, CA 1989). 